\title{
Limit theorem for reflected random walks
}

Hoang-Long Ngo and Marc Peigné

Abstract Let $\xi_{n}, n \in \mathbb{N}$ be a sequence of i.i.d. random variables with values in $\mathbb{Z}$. The associated random walk on $\mathbb{Z}$ is $S(n)=\xi_{1}+\cdots+\xi_{n+1}$ and the corresponding "reflected walk" on $\mathbb{N}_{0}$ is the Markov chain $X=(X(n))_{n \geq 0}$ given by $X(0)=x \in \mathbb{N}_{0}$ and $X(n+1)=\left|X(n)+\xi_{n+1}\right|$ for $n \geq 0$. It is well know that the reflected walk $(X(n))_{n \geq 0}$ is null-recurrent when the $\xi_{n}$ are square integrable and centered. In this paper, we prove that the process $(X(n))_{n \geq 0}$, properly rescaled, converges in distribution towards the reflected Brownian motion on $\mathbb{R}^{+}$, when $\mathbb{E}\left[\xi_{n}^{2}\right]<+\infty, \mathbb{E}\left[\left(\xi_{n}^{-}\right)^{3}\right]<+\infty$ and the $\xi_{n}$ are aperiodic and centered.

\section{Introduction and Notations}

Let $\left(\xi_{n}\right)_{n \geq 1}$ be a sequence of $\mathbb{Z}$-valued, independent and identically distributed random variables, with common law $\mu$ defined on a probability space $(\Omega, \mathcal{F}, \mathbb{P})$. We denote $S=(S(n))_{n \geq 0}$ the classical random walks with steps $\xi_{k}$ defined by $S(0)=0$ and $S(n)=\xi_{1}+\ldots+\xi_{n}$ for any $n \geq 1$.

Throughout this paper, we denote $\mathbb{N}_{0}$ the set of non-negative integers and we consider the reflected random walk $(X(n))_{n \geq 0}$ on $\mathbb{N}_{0}$ defined by

$$
X(n+1)=\left|X(n)+\xi_{n+1}\right|, \quad \text { for } n \geq 0,
$$

where $X(0)$ is a $\mathbb{N}_{0}$-valued random variables. When $X(0)=x \mathbb{P}$-a.s., with $x \in \mathbb{N}_{0}$, the process $(X(n))_{n \geq 0}$ is also denoted by $\left(X^{x}(n)\right)_{n \geq 0}$. It evolves as the random walk

Hoang-Long Ngo

Hanoi National University of Education, 136 Xuan Thuy - Cau Giay - Hanoi - Vietnam, e-mail: ngolong@hnue.edu.vn

Marc Peigné

Institut Denis Poisson UMR 7013, Université de Tours, Université d'Orléans, CNRS France e-mail: peigne@univ-tours.fr 
$x+S(n)$ as long as it stays non negative. When $x+S(n)$ enters the set of negative integers, the sign of its value is changed; the same construction thus applies starting from $|x+S(n)|, \ldots$ and so on.

The process $\left(X^{x}(n)\right)_{n \geq 0}$ is a Markov chain on $\mathbb{N}_{0}$ starting from $x$. Several papers describing its stochastic behavior have been published; we refer to [16] where the recurrence of the reflected random walk is studied under some conditions which are nearly to be optimal. The reader may find also several references therein.

Firstly, $\left(X^{x}(n)\right)_{n \geq 0}$ has some similarities with the classical random walk on $\mathbb{R}$; for instance, a strong law of large numbers holds, namely

$$
\lim _{n \rightarrow+\infty} \frac{X^{x}(n)}{n}=0 \quad \mathbb{P} \text {-a.s. }
$$

when $\mathbb{E}\left[\left|\xi_{n}\right|\right]<+\infty$ and $\mathbb{E}\left[\xi_{n}\right]=0$ (see Lemma 4 in section 3). Nevertheless, in contrast to what holds for the classical random walk on $\mathbb{R}$, this does not yield to the recurrence of $\left(X^{x}(n)\right)_{n \geq 0}$. In [16], it is proved that the process $\left(X^{x}(n)\right)_{n \geq 0}$ is null-recurrent when $\mathbb{E}\left[\left|\xi_{n}\right|^{3 / 2}\right]<+\infty$ and $\mathbb{E}\left[\xi_{n}\right]=0$ and that $\left(X^{x}(n)\right)_{n \geq 0}$ may be transient when $\mathbb{E}\left[\left|\xi_{n}\right|^{3 / 2}\right]=+\infty$, even if $\mathbb{E}\left[\left|\xi_{n}\right|^{3 / 2-\epsilon}\right]<+\infty$ for any $\epsilon>0$. The reader can find in [12] a necessary and sufficient condition for the recurrence of $\left(X^{x}(n)\right)_{n \geq 0}$ (see Theorem 4.6) but this condition cannot be reduced to the existence of some moments.

Once the strong law of large number holds, it is natural to study the oscillations of the process around its expectation. Let us state our result.

Theorem 1 Let $\left(\xi_{n}\right)_{n \geq 1}$ be a sequence of $\mathbb{Z}$-valued i.i.d. random variables such that

A1. $\mathbb{E}\left[\xi_{n}^{2}\right]=\sigma^{2}<+\infty$ and $\mathbb{E}\left[\left(\xi_{n}^{-}\right)^{3}\right]<+\infty \quad(1)$;

A2. $\mathbb{E}\left[\xi_{n}\right]=0$;

A3. The distribution of the $\xi_{n}$ is strongly aperiodic, i.e. the support of the distribution of $\xi_{n}$ is not included in the coset of a proper subgroup of $\mathbb{Z}$.

Let $(X(t))_{t \geq 0}$ be the continuous time process constructed from the sequence $(X(n))_{n \geq 0}$ by linear interpolation between the values at integer points. Then, as $n \rightarrow+\infty$, the sequence of stochastic processes $\left(X_{n}(t)\right)_{n \geq 1}$, defined by

$$
X_{n}(t):=\frac{1}{\sigma \sqrt{n}} X(n t), \quad n \geq 1,0 \leq t \leq 1,
$$

weakly converges in the space of continuous functions on $[0,1]$ to the absolute value $(|B(t)|)_{t \geq 0}$ of the Brownian motion on $\mathbb{R}$.

Let us insist on the fact that $X^{x}(n)$ coincides with $x+S(n)$ as long as it stays non-negative, but after it may differ drastically. The sequence of successive reflection times of $\left(X^{x}(n)\right)_{n \geq 0}$ introduces some strong inhomogeneity on time and makes it necessary to adopt a totally different approach to prove an invariance principle as stated above.

${ }^{1} \xi_{n}^{-}=\max \left(0,-\xi_{n}\right)$ denotes the negative part of $\xi_{n}$ 
A model which is quite similar to $\left(X^{n}(x)\right)_{n \geq 0}$ is the queuing process $\left(W^{x}(n)\right)_{n \geq 0}$, also called the Lindley process, corresponding to the waiting times in a single server queue. We think to $\left(W^{x}(n)\right)_{n \geq 0}$ as an absorbing random walk on $\mathbb{N}_{0}$; as $W^{x}(n)$, it evolves as the random walk $x+S(n)$ as long as it stays non-negative and, when it attempts to cross 0 and become negative, the new value is reset to 0 before continuing. We refer to [15] for precise descriptions and variations on this process and follow the same strategy to obtain the invariance principle.

The excursions of $\left(W^{x}(n)\right)_{n \geq 0}$ and $\left(X^{x}(n)\right)_{n \geq 0}$ between two consecutively times of absorption-reflection coincide with some parts of the trajectory of $(S(n))_{n \geq 0}$, up to a translation; thus, their study is related to the fluctuations of $(S(n))_{n \geq 0}$. Hence, as in [15], we introduce the sequence of strictly descending ladder epochs $\left(\ell_{l}\right)_{l \geq 0}$ of the random walk $(S(n))_{n \geq 0}$ defined inductively by $\ell_{0}=0$ and, for any $l \geq 1$,

$$
\ell_{l+1}:=\min \left\{n>\ell_{l} \mid S(n)<S\left(\ell_{l}\right)\right\} .
$$

When $\mathbb{E}\left[\left|\xi_{n}\right|\right]<+\infty$ and $\mathbb{E}\left[\xi_{n}\right]=0$, the random variables $\ell_{1}, \ell_{2}-\ell_{1}, \ell_{3}-\ell_{2}, \ldots$ are $\mathbb{P}$ a.s. finite and i.i.d. and the same property holds for the random variables $S\left(\ell_{1}\right), S\left(\ell_{2}\right)-$ $S\left(\ell_{1}\right), S\left(\ell_{3}\right)-S\left(\ell_{2}\right), \ldots$. In other words, the processes $\left(\ell_{l}\right)_{l \geq 0}$ and $\left(S\left(\ell_{l}\right)\right)_{l \geq 0}$ are random walks on $\mathbb{N}_{0}$ and $\mathbb{Z}$ with respective distribution $\mathcal{L}\left(\ell_{1}\right)$ and $\mathcal{L}\left(S\left(\ell_{1}\right)\right)$.

Let us briefly point out the main difference between $\left(W^{x}(n)\right)_{n \geq 0}$ and $(X(n))_{n \geq 0}$. At an absorption time, the value of the process $W^{x}(n)$ is reset to 0 before continuing as a classical random walk for a while: there is a total loss of memory of the past after each absorption. Rather, at a reflection time, the process $X^{x}(n)$ equals the absolute value of $x+S(n)$. This value is the "new" starting point of the process, for a while, and has a great influence on the next reflection time; in other words, the process always captures some memory of the past at any time of reflection. This phenomenon has to be taken into account and requires a precise study of the sub-process $\left(X\left(r_{k}\right)\right)_{k \geq 0}$ of $(X(n))_{n \geq 0}$ corresponding to these successive times $\left(r_{k}\right)_{k \geq 0}$ of reflection; our strategy consists in studying the spectrum of the transition probabilities matrix $\mathcal{R}$ of $\left(X\left(r_{k}\right)\right)_{k \geq 0}$, acting on some Banach space $\mathcal{B}=\mathcal{B}_{\alpha}$ of functions from $\mathbb{N}_{0}$ to $\mathbb{C}$ with growth less than $x^{\alpha}$ at infinity, for some $\alpha>0$ to be fixed. In particular, in order to apply recent results on renewal sequences [9], we need precise estimates on the tail of distribution of the reflection times; this is the main reason of the restrictive assumption $\mathbb{E}\left[\left(\xi_{n}^{-}\right)^{3}\right]<+\infty$ instead of moment of order 2, as we could expect. More precisely, throughout the paper, we need the following properties to be satisfied:

(i) The operator $\mathcal{R}$ acts on $\mathcal{B}_{\alpha}$.

This holds when $\mathbb{E}\left[\left|S\left(\ell_{1}\right)\right|^{1+\alpha}\right]<+\infty$ and yields to the condition $\mathbb{E}\left[\left(\xi_{n}^{-}\right)^{2+\alpha}\right]<$ $+\infty$ (see Proposition 1).

(ii) The function $\mathbb{N}_{0} \rightarrow \mathbb{N}_{0}, x \mapsto x$, belongs to $\mathcal{B}_{\alpha}$; this imposes the condition $\alpha \geq 1$ (see Proposition 2).

Eventually, we fix $\alpha=1$ from Section 1 on.

Notations. Throughout the text, we use the following notations. Let $u=\left(u_{n}\right)_{n \geq 0}$ and $v=\left(v_{n}\right)_{n \geq 0}$ be two sequences of positive reals; we write 
- $u \stackrel{c}{\leq} v($ or simply $u \leq v)$ when $u_{n} \leq c v_{n}$ for some constant $c>0$ and $n$ large enough;

- $u_{n} \sim v_{n}$ when $\lim _{n \rightarrow+\infty} \frac{u_{n}}{v_{n}}=1$.

- $u_{n} \approx v_{n}$ when $\lim _{n \rightarrow+\infty}\left(u_{n}-v_{n}\right)=0$.

Acknowledgment H.-L. Ngo thanks the University of Tours for generous hospitality in the Instittue Denis Poisson (IDP) and financial support in May 2019. This article is a result of the research team with the title "Quantitative Research Methods in Economics and Finance", Foreign Trade University, Ha Noi, Vietnam.

M. Peigné thanks the Vietnam Institute for Advanced Studies in Mathematics (VIASM) and the Vietnam Academy of Sciences And Technology (VAST) in Ha Noi for their kind and friendly hospitality and accommodation in June 2018.

Thanks are also due to M. Pollicott who proposed to publish this article in the Chaire Jean Morlet Series.

Both authors thank the referee for many helpful comments that improved the text and some proofs. Duy Tran Vo also pointed them several misprints.

\section{Fluctuations of random walks and auxiliary estimates}

\subsection{On the fluctuation of random walks}

Let $h$ be the Green function of the random walk $\left(S\left(\ell_{l}\right)\right)_{l \geq 0}$, called sometimes the "descending renewal function" of $S$, defined by

$$
h(x)= \begin{cases}\sum_{l=0}^{+\infty} \mathbb{P}\left[S\left(\ell_{l}\right) \geq-x\right] & \text { if } \quad x \geq 0, \\ 0 & \text { otherwise. }\end{cases}
$$

The function $h$ is harmonic for the random walk $(S(n))_{n \geq 0}$ killed when it reaches the negative half line $(-\infty ; 0]$; namely, for any $x \geq 0$,

$$
\mathbb{E}\left[h\left(x+\xi_{1}\right) ; x+\xi_{1}>0\right]=h(x) .
$$

This holds for any oscillating random walk, possible without finite second moment.

Similarly, we denote $\tilde{h}$ the ascending renewal function of the random walk $(S(n))_{n \geq 0}$ (i.e the descending renewal function of $(-S(n))_{n \geq 0}$ ).

Both functions $h$ and $\tilde{h}$ are increasing, $h(0)=\tilde{h}(0)=1$ and $h(x)=O(x), \tilde{h}(x)=$ $O(x)$ as $x \rightarrow+\infty$ (see [2], p. 648).

We have also to take into account the fact that the random walk $S$ does not always start from the origin; hence, for any $x \geq 0$, we set $\tau^{S}(x):=\inf \{n \geq 1: x+S(n)<0\}$; it holds

$$
\left[\tau^{S}(x)>n\right]=\left[L_{n} \geq-x\right]
$$


where $L_{n}=\min (S(1), \ldots, S(n))$. The following result is a combination of Theorem 2 and Proposition 11 in [7] and Theorem A in [13] (see also Theorems II.6 and II.7 in [14]).

Lemma 1 For any $x \geq 0$,

1.

$$
\mathbb{P}\left[\tau^{S}(x)>n\right] \sim c_{1} \frac{h(x)}{\sqrt{n}} \quad \text { as } \quad n \rightarrow+\infty,
$$

where $c_{1}=\frac{\mathbb{E}\left[-S_{\ell_{1}}\right]}{\sigma \sqrt{2 \pi}}$. Moreover, there exists a constant $C_{1}>0$ such that for any $x \geq 0$ and $n \geq 1$,

$$
\mathbb{P}\left[\tau^{S}(x)>n\right] \leq C_{1} \frac{h(x)}{\sqrt{n}}
$$

2. For any $x, y \geq 0$,

$$
\mathbb{P}\left[\tau^{S}(x)>n, x+S(n)=y\right] \sim \frac{1}{\sigma \sqrt{2 \pi}} \frac{h(x) \tilde{h}(y)}{n^{3 / 2}} \quad \text { as } \quad n \rightarrow+\infty,
$$

and there exists a constant $C_{2}>0$ such that, for any any $x, y \geq 0$ and $n \geq 1$,

$$
\mathbb{P}\left[\tau^{S}(x)>n, x+S(n)=y\right] \leq C_{2} \frac{h(x) \tilde{h}(y)}{n^{3 / 2}} .
$$

These assertions yield a precise estimate of the probability $\mathbb{P}\left[\tau^{S}(x)=n\right]$ itself, and not only the tail of the distribution of $\tau^{S}$. As a direct consequence, the sequence of descending ladder epochs $\left(\ell_{l}\right)_{l \geq 1}$ of the random walk $(S(n))_{n \geq 0}$ satisfies some renewal theorem [7]. Let us state these two consequences which enlighten the next section where similar statements concerning the successive epochs of reflections of the reflected random are proved.

Corollary 1 For any $x \geq 0$,

$$
\mathbb{P}\left[\tau^{S}(x)=n\right] \sim \frac{c_{1}}{2} h(x) \frac{1}{n^{3 / 2}} \quad \text { as } \quad n \rightarrow+\infty
$$

and there exists a constant $C_{3}>0$ such that, for any $x \geq 0$ and $n \geq 1$,

$$
\mathbb{P}\left[\tau^{S}(x)=n\right] \leq C_{3} \frac{h(x)}{n^{3 / 2}} .
$$

Furthermore,

$$
\sum_{l=0}^{+\infty} \mathbb{P}\left[\ell_{l}=n\right] \sim \frac{1}{c_{1} \pi} \frac{1}{\sqrt{n}} \quad \text { as } n \rightarrow+\infty
$$




\subsection{Conditional limit theorems}

The following statement corresponds to Lemma 2.3 in [2]; the symbol " $\Rightarrow$ " means "weak convergence".

Lemma 2 Assume $\mathbb{E}\left(\xi_{i}^{2}\right)<+\infty$ and $\mathbb{E}\left(\xi_{i}\right)=0$. Then, for any $x \geq 0$,

$$
\mathcal{L}\left(\left(\frac{S([n t])}{\sigma \sqrt{n}}\right)_{0 \leq t \leq 1} \mid \min \{S(1), \ldots, S(n)\} \geq-x\right) \Rightarrow \mathcal{L}\left(L^{+}\right) \quad \text { as } n \rightarrow+\infty,
$$

where $L^{+}$is the Brownian meander.

In particular, for any bounded and Lipschitz continuous function $\phi: \mathbb{R} \rightarrow \mathbb{R}$,

$$
\lim _{n \rightarrow+\infty} \mathbb{E}\left[\phi\left(\frac{x+S(n)}{\sigma \sqrt{n}}\right) \mid \tau^{S}(x)>n\right]=\int_{0}^{+\infty} \phi(z) z e^{-z^{2} / 2} d z
$$

This Lemma is useful in the sequel to control the fluctuations of the excursions of the process $(X(n))_{n \geq 0}$ between two successive times of reflection. In order to control also the higher dimensional distributions of these excursions, we need some invariance principle for random walk bridges conditioned to stay positive. The following result corresponds in our setting to Corollary 2.5 in [5].

Lemma 3 For any bounded, Lipschitz continuous function $\phi: \mathbb{R} \rightarrow \mathbb{R}$, any $x, y \geq 0$, and any $t>s>0$,

$$
\begin{array}{r}
\lim _{n \rightarrow+\infty} \mathbb{E}\left[\phi\left(\frac{x+S([n s])}{\sigma \sqrt{n}}\right) \mid \tau^{S}(x)>[n t], x+S([n t])=y\right] \\
=\int_{0}^{+\infty} 2 \phi(u \sqrt{s}) \exp \left(-\frac{u^{2}}{2 \frac{s}{t} \frac{t-s}{t}}\right) \frac{u^{2}}{\sqrt{2 \pi \frac{s^{3}}{t^{3}} \frac{(t-s)^{3}}{t^{3}}}} d u .
\end{array}
$$

\section{On the sub-process of reflections}

We present briefly some results from [8] and [16]. The reflected times $r_{n}, n \geq 0$, of the random walk $(X(n))_{n \geq 0}$ are defined by: for any $x \geq 0$,

$$
r_{0}=r_{0}(x)=0 \quad \text { and } \quad r_{n+1}=\inf \left\{m>r_{n} \mid X\left(r_{n}\right)+\xi_{r_{n}+1}+\cdots+\xi_{m}<0\right\} .
$$

Notice that these random variables are $\mathbb{N}_{0} \cup\{+\infty\}$-valued stopping times with respect to the filtration $\left(\mathcal{G}_{n}\right)_{n \geq 0}$.

When $\mathbb{E}\left[\left|\xi_{n}\right|\right]<+\infty$ and $\mathbb{E}\left[\xi_{n}\right]=0$, the random walk $(S(n))_{n \geq 0}$ is oscillating, hence the $r_{n}, n \geq 0$, are all finite $\mathbb{P}$-a.s. and $S(n) / n$ converges $\mathbb{P}$-a.s. towards 0 . The strong law of large numbers is still true for the reflected random walk $\left(X^{x}(n)\right)_{n \geq 0}$ on $\mathbb{N}_{0}$ but does not derive directly. 
Lemma 4 If $\mathbb{E}\left[\left|\xi_{n}\right|\right]<+\infty$ and $\mathbb{E}\left[\xi_{n}\right]=0$, then, for any $x \in \mathbb{N}_{0}$,

$$
\lim _{n \rightarrow+\infty} \frac{X^{x}(n)}{n}=0 \quad \mathbb{P} \text {-a.s. }
$$

Proof For any $n \geq 1$, there exists a (random) integer $k_{n} \geq 1$ such that $r_{k_{n}} \leq n<$ $r_{k_{n}+1}$. It holds

$$
X^{x}(n)=X^{x}\left(r_{k_{n}}\right)+\left(\xi_{r_{k_{n}}+1}+\cdots+\xi_{n}\right)=X^{x}\left(r_{k_{n}}\right)+S(n)-S\left(r_{k_{n}}\right),
$$

so that

$$
0 \leq \frac{X^{x}(n)}{n}=\frac{X^{x}\left(r_{k_{n}}\right)}{n}+\frac{S(n)}{n}-\frac{S\left(r_{k_{n}}\right)}{n} \leq \frac{\max \left\{\left|\xi_{1}\right|, \ldots,\left|\xi_{n}\right|\right\}}{n}+\frac{S(n)}{n}-\frac{S\left(r_{k_{n}}\right)}{n} .
$$

The first term on the right hand side converges $\mathbb{P}$-a.s. towards 0 since $\mathbb{E}\left[\left|\xi_{n}\right|\right]<$ $+\infty$.

By the strong law of large number, the second term tends $\mathbb{P}$-a.s. to 0 .

At last, the same property holds for the last term, since $\left|\frac{S\left(r_{k_{n}}\right)}{n}\right|=\left|\frac{S\left(r_{k_{n}}\right)}{r_{k_{n}}}\right| \times \frac{r_{k_{n}}}{n} \leq$ $\left|\frac{S\left(r_{k_{n}}\right)}{r_{k_{n}}}\right|$.

It follows from Lemma 2.3 in [17] that the sub-process of reflections $\left(X\left(r_{k}\right)\right)_{k \geq 0}$ is a Markov chain on $\mathbb{N}_{0}$ with transition probability $\mathcal{R}$ given by: for all $x, y \in \mathbb{N}_{0}$,

$$
\mathcal{R}(x, y)= \begin{cases}0 & \text { if } y=0 \\ \sum_{w=0}^{x} U^{*}(-w) \mu^{*}(w-x-y) & \text { if } y \geq 1\end{cases}
$$

where $\mu^{*}$ is the distribution of $S\left(\ell_{1}\right)$ and $U^{*}=\sum_{n=0}^{+\infty}\left(\mu^{*}\right)^{\star n}$ denotes its potential.

Set $C:=\sup \{y \geq 1: \mu(-y)>0\}$. The support of $\mu^{*}$ equals $\mathbb{Z}^{-}=\mathbb{Z} \cap(-\infty, 0)$ when $C=+\infty$, otherwise it is $\{-C, \ldots,-1\}$; furthermore, $U^{*}(-w)>0$ for any $w \geq 0$. Then, $\mathcal{R}(x, y)>0$ if and only if $y \in \mathbb{S}_{r}$, where $\mathbb{S}_{r}=\mathbb{N}_{0} \backslash\{0\}$ when $C=+\infty$ and $\mathbb{S}_{r}=\{1, \ldots, C\}$ otherwise. Consequently, the set $\mathbb{S}_{r}$ is the unique irreducible and ergodic class of the Markov chain $\left(X\left(r_{k}\right)\right)_{k \geq 0}$ and this chain is aperiodic on $\mathbb{S}_{r}$.

The measure $v$ on $\mathbb{N}_{0}$ defined by

$$
v(x)=\sum_{y=1}^{+\infty}\left(\frac{1}{2} \mu^{*}(-x)+\mu^{*}((-x-y,-x))+\frac{1}{2} \mu^{*}(-x-y)\right) \mu^{*}(-y),
$$

is, up to a multiplicative constant, the unique stationary measure for $\left(X\left(r_{k}\right)\right)_{k \geq 0}$; its support equals $\mathbb{S}_{r}$ (see Theorem 3.6 [17]).

Notice that this measure $v$ is finite when $\mathbb{E}\left[\xi_{n}\right]=0$ and $\mathbb{E}\left[\left|S\left(\ell_{1}\right)\right|^{1 / 2}\right]<+\infty$ (and in particular when $\mathbb{E}\left[\xi_{n}\right]=0$ and $\left.\mathbb{E}\left[\left|\xi_{n}\right|^{3 / 2}\right]<+\infty[16]\right)$. In this case, we normalize $v$ it in such a way it is a probability measure. 


\subsection{On the spectrum of the transition probabilities matrix $\mathcal{R}$}

Let us recall some spectral properties of the matrix $\mathcal{R}=(\mathcal{R}(x, y))_{x, y \in \mathbb{N}_{0}}$. By Property 2.3 in [8], the matrix $\mathcal{R}$ is quasi-compact on the space $L^{\infty}\left(\mathbb{N}_{0}\right)$ of bounded functions on $\mathbb{N}_{0}$, with 1 as the unique (and simple) dominant eigenvalue; in particular, the rest of the spectrum of $\mathcal{R}$ is included in a disc with radius $<1$.

It is of interest in the next section to let $\mathcal{R}$ act on a bigger space than $L^{\infty}\left(\mathbb{N}_{0}\right)$. For instance, following [8], we may fix $K>1$ and consider the Banach space

$$
L_{K}\left(\mathbb{N}_{0}\right):=\left\{\phi: \mathbb{N}_{0} \rightarrow \mathbb{C}:\|\phi\|_{K}:=\sup _{x \geq 0}|\phi(x)| / K^{x}<+\infty\right\}
$$

endowed with the norm $\|\cdot\|_{K}$. By Property 2.3 in [8], if $\sum_{x \geq 0} K^{x} \mu(x)<+\infty$ then $\mathcal{R}$ acts as a compact operator on $L_{K}\left(\mathbb{N}_{0}\right)$.

In this article, we only assume that $\mu$ has a finite moment of order 2 and its negative part has moment of order 3. Consequently, we consider a smaller Banach space $\mathcal{B}_{\alpha}$ adapted to these hypotheses and defined by: for $\alpha>0$ fixed,

$$
\mathcal{B}_{\alpha}:=\left\{\phi: \mathbb{N}_{0} \rightarrow \mathbb{C}:|\phi|_{\alpha}:=\sup _{x \geq 0} \frac{|\phi(x)|}{1+x^{\alpha}}<+\infty\right\} .
$$

Endowed with the norm $|\cdot|_{\alpha}$, the space $\mathcal{B}_{\alpha}$ is a Banach space on $\mathbb{C}$.

Proposition 1 Fix $\alpha>0$ and assume $\mathbb{E}\left[\xi_{n}^{2}\right]+\mathbb{E}\left[\left(\xi_{n}^{-}\right)^{2+\alpha}\right]<+\infty$ and $\mathbb{E}\left[\xi_{n}\right]=0$. Then, the operator $\mathcal{R}$ acts on $\mathcal{B}_{\alpha}$ and $\mathcal{R}\left(\mathcal{B}_{\alpha}\right) \subset L^{\infty}\left(\mathbb{N}_{0}\right)$. Furthermore,

1. $\mathcal{R}$ is compact on $\mathcal{B}_{\alpha}$ with spectral radius 1 ;

2. 1 is the unique eigenvalue of $\mathcal{R}$ with modulus 1 , it is simple with corresponding eigenspace $\mathbb{C} \mathbf{1}$;

3. the rest of the spectrum of $\mathcal{R}$ on $\mathcal{B}_{\alpha}$ is included in a disc with radius $<1$.

Let $\Pi$ be the projection from $\mathcal{B}_{\alpha}$ onto the eigenspace $\mathbb{C} \mathbf{1}$ corresponding to this spectral decomposition, i.e. such that $\Pi \mathcal{R}=\mathcal{R} \Pi=\Pi$. In other words, there exists a bounded operator $Q$ on $\mathcal{B}_{\alpha}$ with spectral radius $<1$ such that $\mathcal{R}$ may be decomposed as follows:

$$
\mathcal{R}=\Pi+Q, \quad \Pi Q=Q \Pi=0 \quad \text { with } \quad \Pi(\cdot)=v(\cdot) \mathbf{1}
$$

In the next section, we require that $\mathcal{B}_{\alpha}$ does contain the descending and ascending renewal functions $h$ and $\tilde{h}$ of the random walk $S$. This imposes in particular that $\alpha$ is greater or equal to 1 .

Proof (1) By (1), for any $\phi \in \mathcal{B}_{\alpha}$ and $x \geq 0$,

$$
\mathcal{R} \phi(x)=\sum_{y \geq 1} \sum_{w=0}^{x} U^{*}(-w) \mu^{*}(w-x-y) \phi(y)
$$


with $U^{*}(-w)=\sum_{n=0}^{+\infty} \mathbb{P}\left[S\left(l_{n}\right)=-w\right]=\mathbb{P}\left[\cup_{n \geq 0}\left[S\left(l_{n}\right)=-w\right]\right] \leq 1$. Therefore,

$$
\begin{aligned}
|\mathcal{R} \phi(x)| & \leq \sum_{y \geq 1} \sum_{w=0}^{x} \mu^{*}(w-x-y)|\phi(y)| \\
& \leq \sum_{y \geq 1} \mu^{*}((-\infty,-y))|\phi(y)| \\
& \leq\left(\sum_{y \geq 1}\left(1+y^{\alpha}\right) \mu^{*}((-\infty,-y))\right)|\phi|_{\alpha} .
\end{aligned}
$$

By Theorem 1 in [6], the condition $\mathbb{E}\left[\left(\xi_{n}^{-}\right)^{2+\alpha}\right]<+\infty$ implies $\mathbb{E}\left[\left|S\left(\ell_{1}\right)\right|^{1+\alpha}\right]<+\infty$; hence,

$$
\sum_{y \geq 1}\left(1+y^{\alpha}\right) \mu^{*}((-\infty,-y)) \leq \mathbb{E}\left[\left|S\left(\ell_{1}\right)\right|\right]+\mathbb{E}\left[\left|S\left(\ell_{1}\right)\right|^{1+\alpha}\right]<+\infty .
$$

Consequently,

$$
|\mathcal{R} \phi|_{\alpha} \leq|\mathcal{R} \phi|_{\infty} \leq\left(\mathbb{E}\left[\left|S\left(\ell_{1}\right)\right|\right]+\mathbb{E}\left[\left|S\left(\ell_{1}\right)\right|^{1+\alpha}\right]\right)|\phi|_{\alpha}
$$

which proves that $\mathcal{R}$ acts on $\mathcal{B}_{\alpha}$ when $\mathbb{E}\left[\left(\xi_{n}^{-}\right)^{2+\alpha}\right]<+\infty$. More precisely, the operator $\mathcal{R}$ is bounded from $\mathcal{B}_{\alpha}$ into $L^{\infty}\left(\mathbb{N}_{0}\right)$ and since the canonical injection $L^{\infty}\left(\mathbb{N}_{0}\right) \hookrightarrow \mathcal{B}_{\alpha}$ is compact, the operator $\mathcal{R}$ is compact on $\mathcal{B}_{\alpha}$.

Let us now check that $\mathcal{R}$ has spectral radius $\rho_{\alpha}=1$ on $\mathcal{B}_{\alpha}$. On the one hand, the equality $\mathcal{R} \mathbf{1}=\mathbf{1}$, with $\mathbf{1} \in \mathcal{B}_{\alpha}$, yields $\rho_{\alpha} \geq 1$. On the other hands, $\mathcal{R}$ is a power bounded operator on $\mathcal{B}_{\alpha}$, which readily implies $\rho_{\alpha} \leq 1$; indeed, for any $n \geq 1$,

$$
\left|\mathcal{R}^{n} \phi(x)\right| \leq \sum_{z=0}^{+\infty} \mathcal{R}^{n-1}(x, z)|\mathcal{R} \phi(z)| \leq|\mathcal{R} \phi|_{\infty} \sum_{z=0}^{+\infty} \mathcal{R}^{n-1}(x, z)=|\mathcal{R} \phi|_{\infty},
$$

which yields, combining with (2),

$$
\left|\mathcal{R}^{n} \phi\right|_{\alpha} \leq\left|\mathcal{R}^{n} \phi\right|_{\infty} \leq\left(\mathbb{E}\left[\left|S\left(\ell_{1}\right)\right|\right]+\mathbb{E}\left[\left|S\left(\ell_{1}\right)\right|^{1+\alpha}\right]\right)|\phi|_{\alpha}
$$

Consequently, denoting $\left\|\mathcal{R}^{n}\right\|_{\alpha}$ the norm of $\mathcal{R}^{n}$ on $\mathcal{B}_{\alpha}$, it holds

$$
\sup _{n \geq 0}\left\|\mathcal{R}^{n}\right\|_{\alpha} \leq\left(\mathbb{E}\left[\left|S\left(\ell_{1}\right)\right|\right]+\mathbb{E}\left[\left|S\left(\ell_{1}\right)\right|^{1+\alpha}\right]\right)<+\infty .
$$

This achieves the proof of assertion 1.

(2) Let us control the peripherical spectrum of $\mathcal{R}$ in $\mathcal{B}_{\alpha}$. Let $\theta \in \mathbb{R}$ and $\phi \in \mathcal{B}_{\alpha}$ such that $\mathcal{R} \phi=e^{i \theta} \phi$. 
By (2), the function $\mathcal{R} \phi$ is bounded, so is $\phi$. Furthermore, the operator $\mathcal{R}$ being positive, it holds $|\phi| \leq \mathcal{R}|\phi|$. Consequently, the function $|\phi|_{\infty}-|\phi|$ is super-harmonic and non-negative, hence constant since the Markov chain $\left(X\left(r_{n}\right)\right)_{n \geq 0}$ is irreducible and recurrent on this set.

Without loss of generality, we may assume $|\phi|=1$ on $\mathbb{S}_{r}$, i.e $\phi(x)=e^{i \varphi(x)}$ for any $x \in \mathbb{S}_{r}$, with $\varphi: \mathbb{S}_{r} \rightarrow \mathbb{R}$. Equality $\mathcal{R} \phi=e^{i \theta} \phi$ may be rewritten as: for any $x \in \mathbb{S}_{r}$,

$$
\sum_{y \in \mathbb{S}_{r}} e^{i(\varphi(y)-\varphi(x))} \mathcal{R}(x, y)=e^{i \theta}
$$

Recall that $\mathcal{R}(x, y)>0$ for any $x, y \in \mathbb{S}_{r}$; thus, by convexity, $e^{i(\varphi(y)-\varphi(x))}=e^{i \theta}$ for any $x, y \in \mathbb{S}_{r}$. Thus, $e^{i \theta}=1$ and the function $\phi$ is harmonic on $\mathbb{S}_{r}$, hence constant. Eventually, the function $\phi$ is constant on $\mathbb{N}_{0}$ : this is the consequence of equality $\mathcal{R} \phi(x)=e^{i \theta} \phi(x)=\phi(x)$, valid for any $x \in \mathbb{N}_{0}$, combined with the facts that $\mathcal{R}(x, y)>0$ if and only if $y \in \mathbb{S}_{r}$ and that $\phi$ is constant on $\mathbb{S}_{r}$.

(3) Assertion 3 is a consequence of assertion 2 and the compactness of $\mathcal{R}$ on $\mathcal{B}_{\alpha}$.

\subsection{A Renewal limit theorem for the times of reflections}

In this section, we prove the analogous of Corollary 1 for the process $\left(r_{n}\right)_{n \geq 0}$. Let us introduce some notations and conventions.

From now on, we focus on the process $(X(n))_{n \geq 0}$ and denote

$$
\left(\left(\mathbb{N}^{0}\right)^{\otimes \mathbb{N}},\left(\mathcal{P}\left(\mathbb{N}^{0}\right)\right)^{\otimes \mathbb{N}},(X(n))_{n \geq 0},\left(\mathbb{P}_{x}\right)_{x \in \mathbb{N}_{0}}, \theta\right)
$$

the canonical space associated to this process, that is the space of trajectories of the Markov chain $(X(n))_{n \geq 0}$. In particular, $\mathbb{P}_{x}, x \in \mathbb{N}_{0}$, denotes the conditional probability with respect to the event $[X(0)=x]$ and $\mathbb{E}_{x}$ the corresponding conditional expectation. The operator $\theta$ is the classical shift transformation defined by: for any $\left(x_{k}\right)_{k \geq 0} \in\left(\mathbb{N}^{0}\right)^{\otimes \mathbb{N}}$,

$$
\theta\left(\left(x_{k}\right)_{k \geq 0}\right)=\left(\left(x_{k+1}\right)_{k \geq 0} .\right.
$$

For $n \geq 1$ and $x, y \geq 0$, set

$$
R_{n}(x, y):=\mathbb{P}_{x}\left[r_{1}=n, X(n)=y\right]
$$

and

$$
\Sigma_{n}(x, y):=\sum_{k=1}^{+\infty} \mathbb{P}_{x}\left[r_{k}=n, X(n)=y\right] .
$$

We are interested in the behavior as $n \rightarrow+\infty$ of these quantities. It has been already studied in [15] (see Lemma 7) for the Lindley process. For the reflected random walk, the argument is more complicated since the position at time $r_{k}$ may vary, so that the excursions of the random walk $(X(n))_{n \geq 0}$ between two successive reflection 
times are not independent. This explain why we focus here on the reflection process and it is of interest to express quantities $R_{n}(x, y)$ and $\Sigma_{n}(x, y)$ in terms of operators and product of operators related to this sub-process.

We consider the linear operators $R_{n}: L^{\infty}\left(\mathbb{N}_{0}\right) \rightarrow L^{\infty}\left(\mathbb{N}_{0}\right), n \geq 0$, defined by: for any $\phi \in L^{\infty}\left(\mathbb{N}_{0}\right)$ and $x \geq 0$,

$$
R_{n} \phi(x)=\sum_{y \geq 1} R_{n}(x, y) \phi(y)=\mathbb{E}_{x}\left[r_{1}=n ; \phi(X(n))\right] .
$$

In particular, $R_{n}(x, y)=R_{n} \mathbf{1}_{\{y\}}(x)$. The quantity $\Sigma_{n}(x, y)$ is also expressed in terms of the $R_{k}$ as follows:

$$
\begin{aligned}
\Sigma_{n}(x, y) & =\sum_{k=1}^{+\infty} \mathbb{P}_{x}\left[r_{k}=n, X(n)=y\right] \\
& =\sum_{k=1}^{+\infty} \sum_{j_{1}+\cdots+j_{k}=n} \mathbb{P}_{x}\left[r_{1}=j_{1}, r_{2}-r_{1}=j_{2}, \ldots, r_{k}-r_{k-1}=j_{k}, X(n)=y\right] \\
& =\sum_{k=1}^{+\infty} \sum_{j_{1}+\cdots+j_{k}=n} R_{j_{1}} \ldots R_{j_{k}} \mathbf{1}_{\{y\}}(x)
\end{aligned}
$$

Firstly, let us check that the $R_{n}$ act on $\mathcal{B}_{\alpha}$.

Lemma 5 There exists a positive constant $C_{4}$ such that, for any $n \geq 1$ and $\alpha>0$,

$$
\left|R_{n}\right|_{\alpha} \leq C_{4} \frac{\mathbb{E}\left[\left(\xi_{n}^{-}\right)^{2+\alpha}\right]}{n^{3 / 2}} .
$$

Proof For any $\phi \in \mathcal{B}_{\alpha}$ and $x \geq 0$,

$$
\begin{aligned}
\left|R_{n} \phi(x)\right| & \leq \sum_{y \geq 1}|\phi(y)| \mathbb{P}_{x}\left[r_{1}=n, X(n)=y\right] \\
& =\sum_{y \geq 1} \sum_{z \geq 0}|\phi(y)| \mathbb{P}\left[\tau^{S}(x) \geq n-1, x+S(n-1)=z, z+\xi_{n}=-y\right] \\
& =\sum_{y \geq 1} \sum_{z \geq 0}|\phi(y)| \mathbb{P}\left[\tau^{S}(x) \geq n-1, x+S(n-1)=z\right] \mathbb{P}\left[\xi_{n}=-y-z\right] .
\end{aligned}
$$

Hence, by Lemma 1 ,

$$
\frac{\left|R_{n} \phi(x)\right|}{1+x^{\alpha}} \leq \frac{1}{n^{3 / 2}} \sum_{y \geq 1} \sum_{z \geq 0}|\phi(y)| \frac{h(x)}{1+x^{\alpha}} \tilde{h}(z) \mathbb{P}\left[\xi_{1}=-y-z\right] .
$$

Since $h(x)=O(x)$ and $\tilde{h}(z)=O(z)$, 


$$
\begin{aligned}
\frac{\left|R_{n} \phi(x)\right|}{1+x^{\alpha}} & \leq \frac{|\phi|_{\alpha}}{n^{3 / 2}} \sum_{y \geq 1} \sum_{z \geq 0}\left(1+y^{\alpha}\right) \tilde{h}(z) \mathbb{P}\left[\xi_{1}=-y-z\right] \\
& \leq \frac{|\phi|_{\alpha}}{n^{3 / 2}} \sum_{y \geq 1} \sum_{z \geq 0}\left(1+y^{\alpha}\right) z \mathbb{P}\left[\xi_{1}=-y-z\right] \\
& =\frac{|\phi|_{\alpha}}{n^{3 / 2}} \sum_{t \geq 1} \sum_{y=1}^{t}\left(1+y^{\alpha}\right)(t-y) \mathbb{P}\left[\xi_{1}=-t\right] \\
& \leq \frac{|\phi|_{\alpha}}{n^{3 / 2}} \sum_{t \geq 1} t^{2+\alpha} \mathbb{P}\left[\xi_{1}=-t\right]
\end{aligned}
$$

which achieves the proof.

Hence, $\sum_{n \geq 1}\left|R_{n}\right|_{\alpha}<+\infty$; in particular, the sequence $\left(\sum_{n=1}^{N} R_{n}\right)_{N \geq 1}$ converges in $\mathcal{B}_{\alpha}$. Note that its limit equals $\mathcal{R}$ in $\mathcal{B}_{\alpha}$; indeed,

$$
\sum_{n \geq 1} R_{n} \phi(x)=\sum_{n \geq 1} \mathbb{E}_{x}\left[\phi(X(n)), r_{1}=n\right]=\mathbb{E}_{x}\left[\phi\left(X\left(r_{1}\right)\right)\right]=\mathcal{R} \phi(x) .
$$

We can write $\mathcal{R}=\sum_{n \geq 1} R_{n}$ and, for any $z \in \overline{\mathbb{D}}:=\{z \in \mathbb{C}:|z| \leq 1\}$, we set

$$
\mathcal{R}(z)=\sum_{n \geq 1} z^{n} R_{n}
$$

Proposition 2 Fix $\alpha>0$ and assume $\mathbb{E}\left[\xi_{n}^{2}\right]+\mathbb{E}\left[\left(\xi_{n}^{-}\right)^{2+\alpha}\right]<+\infty$ and $\mathbb{E}\left[\xi_{n}\right]=0$. The sequence $\left(R_{n}\right)_{n \geq 0}$ is an aperiodic renewal sequence of operators, i.e. it satisfies the following properties (see [9]):

(R1). The operator $\mathcal{R}=\mathcal{R}(1)$ has a simple eigenvalue at 1 and the rest of its spectrum is contained in a disk of radius $<1$.

(R2). For any $n \geq 1$, set $\mathbf{r}_{n}:=v R_{n} \mathbf{1}=\sum_{x \geq 1} v(x) \mathbb{P}_{x}\left(r_{1}=n\right)$; hence,

$$
\Pi R_{n} \Pi=\mathbf{r}_{n} \Pi
$$

where $\Pi$ denotes the eigenprojection of $\mathcal{R}$ for the eigenvalue 1.

(R3). There exists a constant $\mathbf{C}>0$ such that $\quad\left|R_{n}\right|_{\alpha} \leq \frac{\mathbf{C}}{n^{3 / 2}}$.

(R4). $\sum_{j>n} \mathbf{r}_{j} \sim \frac{\mathbf{c}}{\sqrt{n}}$ with $\mathbf{c}=c_{1} v(h)$, where $c_{1}$ is the positive constant given by Lemma 1 and $h$ is the descending renewal function of the random walk $S$.

(R5). The spectral radius of $\mathcal{R}(z)$ is strictly less than 1 for $z \in \overline{\mathbb{D}} \backslash\{1\}$.

Proof (R1) is a direct consequence of Proposition 1.

(R2) Recall that $\Pi \phi=v(\phi) \mathbf{1}$ for any $\phi \in \mathcal{B}_{\alpha}$. Hence, setting $g_{n}(x):=\mathbb{P}_{x}\left(r_{1}=n\right)$, it holds $R_{n} \Pi \phi=v(\phi) g_{n}$, thus

$$
\Pi R_{n} \Pi \phi=v(\phi) \Pi\left(g_{n}\right)=\sum_{x \geq 1} v(x) \mathbb{P}_{x}\left(r_{1}=n\right) v(\phi) \mathbf{1},
$$


which is the expected result.

(R3) follows from Lemma 5.

(R4) Thanks to Lemma 1,

$$
\sum_{j \geq n} \mathbf{r}_{j}=\sum_{x \geq 1} \sum_{j \geq n} v(x) \mathbb{P}_{x}\left[r_{1}=j\right]=\sum_{x \geq 1} v(x) \mathbb{P}_{x}\left[r_{1} \geq n\right] \sim c_{1} \frac{v(h)}{\sqrt{n}} \quad \text { as } \quad n \rightarrow+\infty .
$$

Notice that $0<v(h)<+\infty$ since $\mathbb{E}\left[\left|S\left(\ell_{1}\right)\right|\right]<+\infty$; indeed, $1 \leq h(x)=O(x)$ and

$$
\begin{aligned}
\sum_{x \geq 1} x v(x) & \leq \sum_{x \geq 1} \sum_{y \geq 1} \sum_{w=x}^{x+y} \mu^{*}(-w) \mu^{*}(-y) x=\sum_{y \geq 1} \sum_{w \geq 1} \mu^{*}(-w) \mu^{*}(-y) \sum_{x=(w-y) \vee 0}^{w} x \\
& \leq \sum_{y \geq 1} \sum_{w \geq 1} y w \mu^{*}(-w) \mu^{*}(-y)=\left(\sum_{y \geq 1} y \mu^{*}(-y)\right)^{2}=\left(\mathbb{E}\left[\left|S\left(\ell_{1}\right)\right|\right]\right)^{2}<+\infty .
\end{aligned}
$$

(R5) The argument is the same as the one used to control the peripherical spectrum of $\mathcal{R}$ in Proposition 1. For any $z \in \overline{\mathbb{D}} \backslash\{1\}$, the operators $\mathcal{R}(z)$ are compact on $\mathcal{B}_{\alpha}$, with spectral radius $\rho_{z} \leq 1$.

If $\rho_{z}=1$, there exist $\theta \in \mathbb{R}$ and $\phi \in \mathcal{B}_{\alpha}$ such that $\mathcal{R}(z) \phi=e^{i \theta} \phi$. Hence $|\phi|=|\mathcal{R}(z) \phi| \leq \mathcal{R}|\phi|$ and since $\mathcal{R}\left(\mathcal{B}_{\alpha}\right) \subset L^{\infty}\left(\mathbb{N}_{0}\right)$, the function $|\phi|$ is bounded on $\mathbb{N}_{0}$, thus constant on $\mathbb{S}_{r}$.

Without loss of generality, we may assume $|\phi|=1$ on $\mathbb{S}_{r}$, i.e $\phi(x)=e^{i \varphi(x)}$ for any $x \in \mathbb{S}_{r}$, with $\varphi: \mathbb{S}_{r} \rightarrow \mathbb{R}$. Equality $\mathcal{R}(z) \phi=e^{i \theta} \phi$ may be rewritten as: for any $x \in \mathbb{S}_{r}$,

$$
\sum_{n \geq 1} \sum_{y \in \mathbb{S}_{r}} z^{n} e^{i \varphi(y)} \mathbb{P}_{x}\left(r_{1}=n ; X(n)=y\right)=e^{i \theta} e^{i \varphi(x)} .
$$

By convexity, since $\sum_{n \geq 1} \sum_{y \in \mathbb{S}_{r}} \mathbb{P}_{x}\left(r_{1}=n ; X(n)=y\right)=1$, we obtain: for all $n \geq 1$ and $x, y \in \mathbb{S}_{r}$,

$$
z^{n} e^{i \varphi(y)}=e^{i \theta} e^{i \varphi(x)}
$$

Setting $x=y$, it yields $z^{n}=e^{i \theta}$, so that $z^{n}$ does not depend on $n$. Finally $z=1$. Thus, $\rho_{z}<1$ when $z \in \overline{\mathbb{D}} \backslash\{1\}$.

By (R5), for $|z|<1$, the operator $T(z):=(I-\mathcal{R}(z))^{-1}$ is well defined in $\mathcal{B}_{\alpha}$; a direct formal computation yields $T(z)=\sum_{n=0}^{+\infty} T_{n} z^{n}$, where the $T_{n}$ are bounded operators on $\mathcal{B}_{\alpha}$ defined by:

$$
T_{0}=I \quad \text { and } \quad T_{n}=\sum_{k=1}^{+\infty} \sum_{j_{1}+\cdots+j_{k}=n} R_{j_{1}} \cdots R_{j_{k}} \quad \text { for } \quad n \geq 1 .
$$

The so-called renewal equation $T(z):=(I-R(z))^{-1}$ is of fundamental importance to understand the asymptotics of the $T_{n}$, several functional analytic tools can be brought into play. Such sequences of operators $\left(R_{n}\right)_{n \geq 0}$ and $\left(T_{n}\right)_{n \geq 0}$ have been the object of many studies, related to renewal theory in a non-commutative setting. We 
refer to the paper [9], which fits perfectly here. The following statement is analogous of the last assertion of Corollary 1 for the reflected random walk.

Corollary 2 The sequence $\left(\sqrt{n} T_{n}\right)_{n \geq 1}$ converges in $\mathcal{B}_{\alpha}$ towards the operator $\frac{1}{\pi c_{1} v(h)} \Pi$.

Proof Apply Theorem 1.4 in [9] with $\beta=1 / 2$ and $\ell(n)=\mathbf{c}=c_{1} v(h)$.

As a direct consequence, by equality (3), it holds

$$
\lim _{n \rightarrow+\infty} \sqrt{n} \Sigma_{n}(x, y)=\frac{v(y)}{\pi c_{1} v(h)}
$$

In the next section, we have to consider and study some modifications of the $\Sigma_{n}(x, y)$ which we introduce now. For any $x \geq 0$ and $0<s<t<1$,

$$
\widehat{\Sigma}_{n}(x, t, s):=n \sum_{l \geq 0} \mathbb{P}_{x}\left[r_{l}=[n s], r_{l+1}>[n t]\right],
$$

and

$$
\widetilde{\Sigma}_{n}(x, t, s):=n^{2} \sum_{l=0}^{+\infty} \mathbb{P}_{x}\left[r_{l}=[n s], r_{l+1}=[n t]\right]
$$

These quantities appear in a natural way to control the finite distribution of the process $\left(X_{n}(t)\right)_{n \geq 0}$.

\section{Proof of Theorem 1}

From now on, we fix $\alpha=1$; this implies that $h \in \mathcal{B}_{\alpha}$, which is necessary from now on (see Lemmas 7 and 9).

\subsection{One-dimensional distribution}

We fix a bounded and Lispchitz continuous function $\phi: \mathbb{R} \rightarrow \mathbb{R}$.

Lemma 6 For any $t \in[0,1]$ and $x \geq 0$, it holds

$$
\lim _{n \rightarrow+\infty} \mathbb{E}_{x}\left[\phi\left(X_{n}(t)\right)\right]=\int_{0}^{+\infty} \phi(u) \frac{2 e^{-u^{2} / 2 t}}{\sqrt{2 \pi t}} d u=\mathbb{E}\left[\phi\left(\left|B_{t}\right|\right)\right],
$$

where $B$ is a standard Brownian motion.

Proof We fix $t \in(0,1)$ and decompose the expectation $\mathbb{E}\left[\phi\left(\frac{X([n t])}{\sigma \sqrt{n}}\right)\right]$ as follows: 


$$
\begin{aligned}
& \mathbb{E}_{x}\left[\phi\left(\frac{X([n t])}{\sigma \sqrt{n}}\right)\right] \\
& \approx \sum_{k=0}^{[n t]-1} \sum_{l \geq 0} \mathbb{E}_{x}\left[\phi\left(\frac{X([n t])}{\sigma \sqrt{n}}\right) ;\right. \\
& \left.r_{l}=k, X(k)+\xi_{k+1} \geq 0, \ldots, X(k)+\xi_{k+1}+\cdots+\xi_{[n t]} \geq 0\right] \\
& =\sum_{k=0}^{[n t]-1} \sum_{y \geq 0} \Sigma_{k}(x, y) \mathbb{E}\left[\phi\left(\frac{y+\xi_{k+1}+\ldots+\xi_{[n t]}}{\sigma \sqrt{n}}\right) ;\right. \\
& =\sum_{k=0}^{[n t]-1} \sum_{y \geq 0} \Sigma_{k}(x, y) \mathbb{E}\left[\phi\left(\frac{y+S([n t]-k)}{\sigma \sqrt{n}}\right) \mid \tau^{S}(y)>[n t]-k\right] \\
& \times \mathbb{P}\left[\tau^{S}(y)>[n t]-k\right] .
\end{aligned}
$$

For each $k=2, \ldots,[n t]-4$ and any $s \in\left[\frac{k}{n}, \frac{k+1}{n}\right)$,

$$
\begin{aligned}
f_{n}(s)=n \sum_{y \geq 0} \Sigma_{[n s]}(x, y) \mathbb{E}[ & \left.\phi\left(\frac{y+S([n t]-[n s])}{\sigma \sqrt{n}}\right) \mid \tau^{S}(y)>[n t]-[n s]\right] \\
& \times \mathbb{P}\left[\tau^{S}(y)>[n t]-[n s]\right],
\end{aligned}
$$

and $f_{n}(s)=0$ on $\left[0, \frac{2}{n}\right)$ and $\left[\frac{[n t]-1}{n}, t\right)$. Hence,

$$
\mathbb{E}_{x}\left[\phi\left(\frac{X([n t])}{\sigma \sqrt{n}}\right)\right]=\int_{0}^{t} f_{n}(s) d s+O\left(\frac{1}{\sqrt{n}}\right) .
$$

Now, let us set : for $n \geq 1$ and any $y \in \mathbb{N}_{0}$,

$$
\begin{aligned}
& a_{n}(y)=\Sigma_{[n s]}(x, y) \mathbb{P}\left[\tau^{S}(y)>[n t]-[n s]\right], \\
& b_{n}(y)=\mathbb{E}\left[\phi\left(\frac{y+S([n t]-[n s])}{\sigma \sqrt{n}}\right) \mid \tau^{S}(y)>[n t]-[n s]\right] .
\end{aligned}
$$

For any $n \geq 1$, it holds

$$
\sum_{y \geq 0} a_{n}(y)=n \sum_{l \geq 0} \mathbb{P}_{x}\left[r_{l}=[n s], r_{l+1}>[n t]\right]=: \widehat{\Sigma}_{n}(x, t, s),
$$

and $\left|b_{n}(y)\right| \leq|\phi|_{\infty}$. The two following lemmas allow us to control the behavior as $n \rightarrow+\infty$ of the integral $\int_{0}^{t} f_{n}(s) d s$; the proof of Lemma 7 is postponed to the last section, the one of 8 is straightforward. 
Lemma 7 For each $0<s<t<1$,

$$
\lim _{n \rightarrow+\infty} \widehat{\Sigma}_{n}(x, t, s)=\frac{1}{\pi \sqrt{s(t-s)}} .
$$

Moreover, there exists a positive constant $C_{5}$ such that

$$
\widehat{\Sigma}_{n}(x, t, s) \leq C_{5} \frac{1+x}{\sqrt{s(t-s)}} \text { for all } 0<s<t<1 \text { and } x \in \mathbb{N} .
$$

Lemma 8 Let $\left(a_{n}(y)\right)_{y \in \mathbb{N}_{0}^{k}},\left(b_{n}(y)\right)_{y \in \mathbb{N}_{0}^{k}}$ be arrays of real numbers for some integer $k \geq 1$. Suppose that

- $a_{n}(y) \geq 0$

- $\lim _{n \rightarrow+\infty} \sum_{y \in \mathbb{N}_{0}^{k}} a_{n}(y)=A$;

- $\lim _{n \rightarrow+\infty} b_{n}(y)=B$ for all $y \in \mathbb{N}_{0}^{k}$;

- $\sup _{n \geq 1, y \in \mathbb{N}^{k}}\left|b_{n}(y)\right|<+\infty$.

Then

$$
\lim _{n \rightarrow+\infty} \sum_{y \geq 0} a_{n}(y) b_{n}(y)=A B .
$$

Lemmas 2, 7 and 8 combined altogether yield: for any $s \in(0, t)$,

$$
\lim _{n \rightarrow+\infty} f_{n}(s)=\frac{1}{\pi} \frac{1}{\sqrt{s(t-s)}} \int_{0}^{+\infty} \phi(z \sqrt{t-s}) z e^{-z^{2} / 2} d z .
$$

Moreover,

$$
\sup _{n}\left|f_{n}(s)\right| \leq C_{5} \frac{1+x}{\sqrt{s(t-s)}}|\phi|_{\infty}=: \hat{f}(s) .
$$

Since $\hat{f} \in L^{1}[0, t]$, the Lebesgue dominated convergence theorem yields

$$
\begin{aligned}
\lim _{n \rightarrow+\infty} \mathbb{E}\left[\phi\left(\frac{X([n t])}{\sigma \sqrt{n}}\right)\right] & =\lim _{n \rightarrow+\infty} \int_{0}^{t} f_{n}(s) d s \\
& =\frac{1}{\pi} \int_{0}^{t} \frac{1}{\sqrt{s(t-s)}}\left(\int_{0}^{+\infty} \phi(z \sqrt{t-s}) z e^{-z^{2} / 2} d z\right) d s \\
& =\int_{0}^{+\infty} \phi(u) \frac{2 e^{-u^{2} / 2 t}}{\sqrt{2 \pi t}} d u,
\end{aligned}
$$

where the last equation follows from the identity ([11], p. 17)

$$
\int_{0}^{+\infty} \frac{1}{\sqrt{t}} \exp \left(-\alpha t-\frac{\beta}{t}\right) d t=\sqrt{\frac{\pi}{\alpha}} e^{-2 \sqrt{\alpha \beta}} \quad(\alpha, \beta>0)
$$


and some change of variable computation. We achieve the proof of Lemma 6 by noting that, since $\phi$ is Lipschitz continuous (with Lipschitz coefficient $[\phi]$ ),

$$
\begin{aligned}
\left|\mathbb{E}_{x}\left[\phi\left(\frac{X([n t])}{\sigma \sqrt{n}}\right)\right]-\mathbb{E}_{x}\left[\phi\left(X_{n}(t)\right)\right]\right| & \leq[\phi] \mathbb{E}_{x}\left[\left|\frac{X([n t])}{\sigma \sqrt{n}}-X_{n}(t)\right|\right] \\
& \leq \frac{1}{\sigma \sqrt{n}}[\phi] \mathbb{E}\left[\left|\xi_{[n t]+1}\right|\right] \rightarrow 0 \text { as } n \rightarrow+\infty .
\end{aligned}
$$

\subsection{Two-dimensional distributions}

The convergence of the finite-dimensional distributions of $\left(X_{n}(t)\right)_{n \geq 1}$ is more delicate. We detail the argument for two-dimensional ones, the general case may be treated in a similar way.

Let us fix $0<s<t, n \geq 1$ and denote

$$
\kappa=\kappa(n, s)=\min \left\{k>[n s]: X(k-1)+\xi_{k}<0\right\} .
$$

We decompose $\mathbb{E}_{x}\left[\phi_{1}\left(\frac{X([n s])}{\sigma \sqrt{n}}\right) \phi_{2}\left(\frac{X([n t])}{\sigma \sqrt{n}}\right)\right]$ as

$$
\begin{aligned}
\underbrace{\sum_{k=[n s]+1}^{[n t]} \mathbb{E}_{x}\left[\phi_{1}\left(\frac{X([n s])}{\sigma \sqrt{n}}\right) \phi_{2}\left(\frac{X([n t])}{\sigma \sqrt{n}}\right) \mathbf{1}_{\{k=k\}}\right]}_{A_{1}(n)} \\
+\underbrace{\underbrace{}_{\mathbb{E}_{X}\left[\phi_{1}\left(\frac{X([n s])}{\sigma \sqrt{n}}\right) \phi_{2}\left(\frac{X([n t])}{\sigma \sqrt{n}}\right) \mathbf{1}_{\{\kappa>[n t]\}}\right]}}_{A_{2}(n)} .
\end{aligned}
$$

The term $A_{1}(n)$ deals with the trajectories of the process $X$ which reflect between $[n s]+1$ and $[n t]$ while $A_{2}(n)$ concerns the others trajectories.

\subsubsection{Estimate of $A_{1}(n)$}

As in the previous section, we decompose $A_{1}(n)$ as 


$$
\begin{aligned}
& A_{1}(n)=\sum_{k_{1}=0}^{[n s]-1} \sum_{k_{2}=[n s]}^{[n t]} \sum_{l=0}^{+\infty} \sum_{y \geq 1} \sum_{z \geq 1} \sum_{w \geq 0} \\
& \mathbb{E}_{x}\left[\phi_{1}\left(\frac{X([n s])}{\sigma \sqrt{n}}\right) \phi_{2}\left(\frac{X([n t])}{\sigma \sqrt{n}}\right) ;\right. \\
& r_{l}=k_{1}, X\left(k_{1}\right)=z, z+\xi_{k_{1}+1} \geq 0, \ldots, z+\xi_{k_{1}+1}+\cdots+\xi_{k_{2}-2} \geq 0, \\
& \left.z+\xi_{k_{1}+1}+\cdots+\xi_{k_{2}-1}=w, w+\xi_{k_{2}}=-y\right] \\
& =\sum_{k_{1}=0}^{[n s]-1} \sum_{k_{2}=[n s]}^{[n t]} \sum_{l=0}^{+\infty} \sum_{y \geq 1} \sum_{z \geq 1} \sum_{w \geq 0} \mathbb{E}_{x}\left[\phi_{1}\left(\frac{z+\xi_{k_{1}+1}+\cdots+\xi_{[n s]}}{\sigma \sqrt{n}}\right)\right. \\
& \times \phi_{2}\left(\frac{y+\xi_{k_{2}+1}+\cdots+\xi_{[n t]}}{\sigma \sqrt{n}}\right) \text {; } \\
& r_{l}=k_{1}, X\left(k_{1}\right)=z, z+\xi_{k_{1}+1} \geq 0, \ldots, z+\xi_{k_{1}+1}+\cdots+\xi_{k_{2}-2} \geq 0 \text {, } \\
& \left.z+\xi_{k_{1}+1}+\cdots+\xi_{k_{2}-1}=w, w+\xi_{k_{2}}=-y\right] \\
& =\sum_{k_{1}=0}^{[n s]-1} \sum_{k_{2}=[n s]}^{[n t]} \sum_{l=0}^{+\infty} \sum_{y \geq 1} \sum_{z \geq 1} \sum_{w \geq 0} \\
& \mathbb{E}_{x}\left[\phi_{2}\left(\frac{y+\xi_{k_{2}+1}+\cdots+\xi_{[n t]}}{\sigma \sqrt{n}}\right)\right] \mathbb{P}_{x}\left[r_{l}=k_{1}, X\left(k_{1}\right)=z\right] \\
& \times \mathbb{E}_{x}\left[\phi_{1}\left(\frac{z+\xi_{k_{1}+1}+\cdots+\xi_{[n s]}}{\sigma \sqrt{n}}\right),\right. \\
& z+\xi_{k_{1}+1} \geq 0, \ldots, z+\xi_{k_{1}+1}+\cdots+\xi_{k_{2}-2} \geq 0 \\
& \left.z+\xi_{k_{1}+1}+\cdots+\xi_{k_{2}-1}=w, w+\xi_{k_{2}}=-y\right] \text {. }
\end{aligned}
$$

Using the fact that the $\xi_{k}$ are i. i. d., we obtain

$$
\begin{aligned}
A_{1}(n) & =\sum_{k_{1}=0}^{[n s]-1} \sum_{z \geq 1} \Sigma_{k_{1}}(x, z) \sum_{k_{2}=[n s]}^{[n t]} \sum_{y \geq 1} \sum_{w \geq 0} \mathbb{E}_{y}\left[\phi_{2}\left(\frac{X\left([n t]-k_{2}\right)}{\sigma \sqrt{n}}\right)\right] \\
& \times \mathbb{E}\left[\phi_{1}\left(\frac{z+S\left([n s]-k_{1}\right)}{\sigma \sqrt{n}}\right) \mid \tau^{S}(z)>k_{2}-k_{1}-1, z+S\left(k_{2}-k_{1}-1\right)=w\right] \\
& \times \mathbb{P}\left[\tau^{S}(z)>k_{2}-k_{1}-1, z+S\left(k_{2}-k_{1}-1\right)=w\right] \mathbb{P}\left[\xi_{1}=-w-y\right] .
\end{aligned}
$$

For any $2 \leq k_{1}<[n s]-6$ and $[n s] \leq k_{2} \leq[n t]$ and any $s_{1} \in\left[\frac{k_{1}}{n}, \frac{k_{1}+1}{n}\right)$ and $s_{2} \in\left[\frac{k_{2}}{n}, \frac{k_{2}+1}{n}\right)$, we write 


$$
\begin{aligned}
& f_{n}\left(s_{1}, s_{2}\right)=n^{2} \sum_{z \geq 1} \Sigma_{\left[n s_{1}\right]}(x, z) \sum_{y \geq 1} \sum_{w \geq 0} \mathbb{E}_{y}\left[\phi_{2}\left(\frac{X\left([n t]-\left[n s_{2}\right]\right)}{\sigma \sqrt{n}}\right)\right] \\
& \times \mathbb{E}\left[\phi_{1}\left(\frac{z+S\left([n s]-\left[n s_{1}\right]\right)}{\sigma \sqrt{n}}\right) \mid \tau^{S}(z)>\left[n s_{2}\right]-\left[n s_{1}\right]-1,\right. \\
& \left.z+S\left(\left[n s_{2}\right]-\left[n s_{1}\right]-1\right)=w\right] \\
& \times \mathbb{P}\left[\tau^{S}(z)>\left[n s_{2}\right]-\left[n s_{1}\right]-1, z+S\left(\left[n s_{2}\right]-\left[n s_{1}\right]-1\right)=w\right] \mathbb{P}\left[\xi_{1}=-w-y\right],
\end{aligned}
$$

and $f_{n}\left(s_{1}, s_{2}\right)=0$ for the others values of $k_{1}$, such that $0 \leq k_{1} \leq[n s]$. Hence,

$$
A_{1}(n)=\int_{0}^{s} d s_{1} \int_{s}^{t} d s_{2} f_{n}\left(s_{1}, s_{2}\right)+O\left(\frac{1}{\sqrt{n}}\right) .
$$

It follows from Lemma 3 that, for each $z, w \geq 0$,

$$
\begin{gathered}
\lim _{n \rightarrow+\infty} \mathbb{E}\left[\phi_{1}\left(\frac{z+S\left([n s]-\left[n s_{1}\right]\right)}{\sigma \sqrt{n}}\right) \mid \tau^{S}(z)>\left[n s_{2}\right]-\left[n s_{1}\right]-1,\right. \\
\left.z+S\left(\left[n s_{2}\right]-\left[n s_{1}\right]-1\right)=w\right] \\
=\int_{0}^{+\infty} 2 \phi_{1}\left(u \sqrt{s_{2}-s_{1}}\right) \exp \left(-\frac{u^{2}}{2 \frac{s-s_{1}}{s_{2}-s_{1}} \frac{s_{2}-s}{s_{2}-s_{1}}}\right) \frac{u^{2}}{\sqrt{2 \pi \frac{\left(s-s_{1}\right)^{3}}{\left(s_{2}-s_{1}\right)^{3}}} \frac{\left(s_{2}-s\right)^{3}}{\left(s_{2}-s_{1}\right)^{3}}} d u \\
=\frac{2}{\sqrt{2 \pi}} \int_{0}^{+\infty} \phi_{1}(v) \exp \left(-\frac{v^{2}}{2 \frac{\left(s-s_{1}\right)\left(s_{2}-s\right)}{s_{2}-s_{1}}}\right) \frac{v^{2}}{\sqrt{\frac{\left(s-s_{1}\right)^{3}\left(s_{2}-s\right)^{3}}{\left(s_{2}-s_{1}\right)^{3}}}} d v .
\end{gathered}
$$

By Lemma 6,

$$
\lim _{n \rightarrow+\infty} \mathbb{E}_{y}\left[\phi_{2}\left(\frac{X\left([n t]-\left[n s_{2}\right]\right)}{\sigma \sqrt{n}}\right)\right]=\int_{0}^{+\infty} \phi_{2}(u) \frac{2 e^{-u^{2} / 2\left(t-s_{2}\right)}}{\sqrt{2 \pi\left(t-s_{2}\right)}} d u .
$$

We set 


$$
\begin{aligned}
& a_{n}(x, y, z, w)= n^{2} \Sigma_{\left[n s_{1}\right]}(x, z) \\
& \times \mathbb{P}\left[\tau^{S}(z)>\left[n s_{2}\right]-\left[n s_{1}\right]-1, z+S\left(\left[n s_{2}\right]-\left[n s_{1}\right]-1\right)=w\right] \\
& \times \mathbb{P}\left[\xi_{1}=-w-y\right], \\
& b_{n}(y, z, w)= \mathbb{E}_{y}\left[\phi_{2}\left(\frac{X\left([n t]-\left[n s_{2}\right]\right)}{\sigma \sqrt{n}}\right)\right] \\
& \times \mathbb{E}\left[\phi_{1}\left(\frac{z+S\left([n s]-\left[n s_{1}\right]\right)}{\sigma \sqrt{n}}\right) \mid \tau^{S}(z)>\left[n s_{2}\right]-\left[n s_{1}\right]-1,\right. \\
&\left.z+S\left(\left[n s_{2}\right]-\left[n s_{1}\right]-1\right)=w\right]
\end{aligned}
$$

Note that $\sum_{z \geq 1} \sum_{y \geq 1} \sum_{w \geq 0} a_{n}(x, y, z, w)=\widetilde{\Sigma}_{n}\left(x, s_{2}, s_{1}\right)$. The behavior as $n \rightarrow+\infty$ of the quantity $\widetilde{\Sigma}_{n}\left(x, s_{2}, s_{1}\right)$ is given by the following Lemma, whose proof is postponed to the last section.

Lemma 9 For all $0<s<t<1$, it holds

$$
\lim _{n \rightarrow+\infty} \widetilde{\Sigma}_{n}(x, t, s)=\frac{1}{2 \pi \sqrt{s(t-s)^{3}}} .
$$

Moreover, there exists a positive constant $C_{6}$ such that, for all $0<s<t<1$ and $n \geq 0$,

$$
\widetilde{\Sigma}_{n}(x, t, s) \leq C_{6} \frac{1+x}{\pi \sqrt{s(t-s)^{3}}} .
$$

By Lemmas 9 and 8 , we get $\lim _{n \rightarrow+\infty} f_{n}\left(s_{1}, s_{2}\right)=f\left(s_{1}, s_{2}\right)$ where

$$
\begin{aligned}
f\left(s_{1}, s_{2}\right)= & \frac{1}{\pi^{2} \sqrt{s_{1}}} \int_{0}^{+\infty} \phi_{1}(v) \exp \left(-\frac{v^{2}}{2 \frac{\left(s_{2}-s\right)\left(s-s_{1}\right)}{s_{2}-s_{1}}}\right) \frac{v^{2}}{\sqrt{\left(s-s_{1}\right)^{3}\left(s_{2}-s\right)^{3}}} d v \\
& \times \int_{0}^{+\infty} \phi_{2}(u) \frac{e^{-u^{2} / 2\left(t-s_{2}\right)}}{\sqrt{t-s_{2}}} d u .
\end{aligned}
$$

Moreover, following the argument in the proof of Lemma 6, we can show that the sequence $\left(\left|f_{n}\right|\right)_{n \geq 1}$ is uniformly bounded by a function which is integrable with respect to Lebesgue measure on $[0, s] \times[s, t]$. Hence, using again the Lebesgue dominated convergence theorem, we get 


$$
\begin{aligned}
& \lim _{n \rightarrow+\infty} A_{1}(n)=\int_{0}^{s} d s_{1} \int_{s}^{t} d s_{2} f\left(s_{1}, s_{2}\right) \\
&=\frac{1}{\pi^{2}} \int_{0}^{s} \frac{d s_{1}}{\sqrt{s_{1}}} \int_{s}^{t} d s_{2} \int_{0}^{+\infty} \phi_{1}(v) \exp \left(-\frac{v^{2}}{2 \frac{\left(s_{2}-s\right)\left(s-s_{1}\right)}{s_{2}-s_{1}}}\right) \frac{v^{2}}{\sqrt{\left(s-s_{1}\right)^{3}\left(s_{2}-s\right)^{3}}} \\
& \times \int_{0}^{+\infty} \phi_{2}(u) \frac{e^{-u^{2} / 2\left(t-s_{2}\right)}}{\sqrt{t-s_{2}}} d u d v,
\end{aligned}
$$

which yields, using again (4),

$$
\lim _{n \rightarrow+\infty} A_{1}(n)=\frac{2}{\pi \sqrt{s(t-s)}} \int_{0}^{+\infty} \int_{0}^{+\infty} \phi_{1}(v) \phi_{2}(u) e^{-v^{2} / 2 s} e^{-\frac{(u+v)^{2}}{2(t-s)}} d u d v .
$$

\subsubsection{Estimate of $A_{2}(n)$}

We decompose $A_{2}(n)$ as

$$
\begin{aligned}
\sum_{y=0}^{+\infty} \sum_{k \leq[n s]} \sum_{l \geq 0} \mathbb{E}_{x}\left[\phi_{1}\left(\frac{X([n s])}{\sigma \sqrt{n}}\right) \phi_{2}\left(\frac{X([n t])}{\sigma \sqrt{n}}\right) ;\right. \\
\left.r_{l}=k, X(k)=y, y+\xi_{k+1} \geq 0, \ldots, y+\xi_{k+1}+\cdots+\xi_{[n t]} \geq 0\right] \\
=\sum_{y=0}^{+\infty} \sum_{k \leq[n s]} \mathbb{E}_{x}\left[\phi_{1}\left(\frac{y+\xi_{k+1}+\cdots+\xi_{[n s]}}{\sigma \sqrt{n}}\right) \phi_{2}\left(\frac{y+\xi_{k+1}+\cdots+\xi_{[n t]}}{\sigma \sqrt{n}}\right) ;\right. \\
\left.y+\xi_{k+1} \geq 0, \ldots, y+\xi_{k+1}+\cdots+\xi_{[n t]} \geq 0\right] \\
\times \sum_{l \geq 0} \mathbb{P}_{x}\left[r_{l}=k, X(k)=y\right] .
\end{aligned}
$$

Since $\left(\xi_{k}\right)$ is a i.i.d. sequence,

$$
\begin{aligned}
& A_{2}(n)=\sum_{y=0}^{+\infty} \sum_{k \leq[n s]} \Sigma_{k}(x, y) \mathbb{E}\left[\phi_{1}\left(\frac{y+S([n s]-k)}{\sigma \sqrt{n}}\right) \phi_{2}\left(\frac{y+S([n t]-k)}{\sigma \sqrt{n}}\right)\right. \\
&\left.\tau^{S}(y)>[n t]-k\right] .
\end{aligned}
$$

For $u \in(0, s]$, we denote 


$$
\begin{aligned}
& g_{n}(u)=n \sum_{y=0}^{+\infty} \Sigma_{[n u]}(x, y) \mathbb{E}\left[\phi_{1}\left(\frac{y+S([n s]-[n u])}{\sigma \sqrt{n}}\right) \phi_{2}\left(\frac{y+S([n t]-[n u])}{\sigma \sqrt{n}}\right) ;\right. \\
& \left.\tau^{S}(y)>[n t]-[n u]\right] .
\end{aligned}
$$

Now, let us compute the pointwise limit on $(0, s]$ of the sequence $\left(g_{n}\right)_{n \geq 1}$. We write $g_{n}(u)$ as

$$
\begin{aligned}
& g_{n}(u)=n \sum_{y=0}^{+\infty} \Sigma_{[n u]}(x, y) \\
& \quad \times \mathbb{E}\left[\phi_{1}\left(\frac{y+S([n s]-[n u])}{\sigma \sqrt{n}}\right) \phi_{2}\left(\frac{y+S([n t]-[n u])}{\sigma \sqrt{n}}\right) \mid \tau^{S}(y)>[n t]-[n u]\right] \\
& \times \mathbb{P}_{y}\left[\tau^{S}(y)>[n t]-[n u]\right] .
\end{aligned}
$$

We set

$$
a_{n}(x, y)=n \Sigma_{[n u]}(x, y) \mathbb{P}_{y}\left[\tau^{S}(y)>[n t]-[n u]\right],
$$

and

$$
b_{n}(y)=\mathbb{E}\left[\phi_{1}\left(\frac{y+S([n s]-[n u])}{\sigma \sqrt{n}}\right) \phi_{2}\left(\frac{y+S([n t]-[n u])}{\sigma \sqrt{n}}\right) \mid \tau^{S}(y)>[n t]-[n u]\right] .
$$

Note that $\sum_{y=0}^{+\infty} a_{n}(y)=\widehat{\Sigma}_{[n u]}(x, t, u)$. Since $\phi_{1}, \phi_{2}$ are bounded and continuous on $\mathbb{R}$, it follows from Theorem 3.2 in [4] and Theorems 2.23 and 3.4 in [10] that

$$
\begin{aligned}
\lim _{n \rightarrow+\infty} b_{n}(y)= & \lim _{n \rightarrow+\infty} \mathbb{E}\left[\phi_{1}\left(\frac{y+S([n s]-[n u])}{\sigma \sqrt{[n t]-[n u]}} \frac{\sqrt{[n t]-[n u]}}{\sqrt{n}}\right)\right. \\
& \left.\times \phi_{2}\left(\frac{y+S([n t]-[n u])}{\sigma \sqrt{[n t]-[n u]}} \frac{\sqrt{[n t]-[n u]}}{\sqrt{n}}\right) \mid \tau^{S}(y)>[n t]-[n u]\right] \\
= & \int_{0}^{+\infty} \int_{0}^{+\infty} \phi_{1}(y \sqrt{t-u}) \phi_{2}(z \sqrt{t-u})\left(\frac{t-u}{s-u}\right)^{3 / 2} y e^{-\frac{t-u}{2(s-u)} y^{2}} \\
& \times \frac{e^{-\frac{1}{2} \frac{t-u}{t-s}(z-y)^{2}}-e^{-\frac{1}{2} \frac{t-u}{t-s}(z+y)^{2}}}{\sqrt{2 \pi\left(1-\frac{s-u}{t-u}\right)}} d y d z \\
= & \frac{1}{\sqrt{2 \pi(t-s)}} \int_{0}^{+\infty} \int_{0}^{+\infty} \phi_{1}\left(y^{\prime}\right) \phi_{2}\left(z^{\prime}\right) \frac{\sqrt{t-u}}{(s-u)^{3 / 2}} y^{\prime} e^{-\frac{y^{\prime 2}}{2(s-u)}} \\
& \times\left(e^{-\frac{\left(z^{\prime}-y^{\prime}\right)^{2}}{2(t-s)}}-e^{-\frac{\left(z^{\prime}+y^{\prime}\right)^{2}}{2(t-s)}}\right) d y^{\prime} d z^{\prime} .
\end{aligned}
$$


Again, we can use the argument in the proof of Lemma 6 to show that the sequence $\left(g_{n}\right)$ converges point wise to $g$ with

$$
\begin{aligned}
& g(u)=\frac{1}{\pi^{3 / 2} \sqrt{2(t-s)}} \frac{1}{\sqrt{u(s-u)^{3}}} \\
& \times \int_{0}^{+\infty} \int_{0}^{+\infty} \phi_{1}\left(y^{\prime}\right) \phi_{2}\left(z^{\prime}\right) y^{\prime} e^{-\frac{y^{\prime 2}}{2(s-u)}}\left(e^{-\frac{\left(z^{\prime}-y^{\prime}\right)^{2}}{2(t-s)}}-e^{-\frac{\left(z^{\prime}+y^{\prime}\right)^{2}}{2(t-s)}}\right) d y^{\prime} d z^{\prime}
\end{aligned}
$$

and $\left(g_{n}\right)$ is also dominated by a function which is integrable on $[0, s]$ with respect to the Lebesgue measure. Lebesgue's dominated convergence theorem yields

$$
\begin{aligned}
& \lim _{n \rightarrow+\infty} A_{2}(n)=\lim _{n \rightarrow+\infty} \frac{1}{n} \sum_{k \leq[n s]} g_{n}(k / n)=\int_{0}^{s} g(u) d u \\
& =\frac{1}{\pi^{3 / 2} \sqrt{2(t-s)}} \int_{0}^{s} d u \int_{0}^{+\infty} d y^{\prime} \int_{0}^{+\infty} d z^{\prime} \\
& \quad \times \phi_{1}\left(y^{\prime}\right) \phi_{2}\left(z^{\prime}\right) \frac{e^{-\frac{y^{\prime 2}}{2(s-u)}}}{\sqrt{u(s-u)^{3}}} \frac{y^{\prime}}{\sqrt{2 \pi(t-s)}}\left(e^{-\frac{\left(z^{\prime}-y^{\prime}\right)^{2}}{2(t-s)}}-e^{-\frac{\left(z^{\prime}+y^{\prime}\right)^{2}}{2(t-s)}}\right) \\
& =\frac{1}{\pi^{3 / 2} s \sqrt{2(t-s)}} \int_{0}^{+\infty} d y^{\prime} \int_{0}^{+\infty} d z^{\prime} \phi_{1}\left(y^{\prime}\right) \phi_{2}\left(z^{\prime}\right)\left(e^{-\frac{\left(z^{\prime}-y^{\prime}\right)^{2}}{2(t-s)}}-e^{-\frac{\left(z^{\prime}+y^{\prime}\right)^{2}}{2(t-s)}}\right) \\
& \quad \times\left(\int_{0}^{1} \frac{y^{\prime}}{\sqrt{v(1-v)^{3}}} e^{-\frac{y^{\prime 2}}{2 s(1-v)}} d v\right) \\
& =\frac{1}{\pi \sqrt{s(t-s)}} \int_{0}^{+\infty} d y^{\prime} \int_{0}^{+\infty} d z^{\prime} \phi_{1}\left(y^{\prime}\right) \phi_{2}\left(z^{\prime}\right) e^{-y^{\prime 2} / 2 s}\left(e^{-\frac{\left(z^{\prime}-y^{\prime}\right)^{2}}{2(t-s)}}-e^{-\frac{\left(z^{\prime}+y^{\prime}\right)^{2}}{2(t-s)}}\right) .
\end{aligned}
$$

\subsubsection{Conclusion}

Combining (6) and (7), we may write

$$
\begin{aligned}
\lim _{n \rightarrow+\infty} & \mathbb{E}\left[\phi_{1}\left(\frac{X([n s])}{\sigma \sqrt{n}}\right) \phi_{2}\left(\frac{X([n t])}{\sigma \sqrt{n}}\right)\right] \\
& =\frac{1}{\pi \sqrt{s(t-s)}} \int_{0}^{+\infty} d y^{\prime} \int_{0}^{+\infty} d z^{\prime} \phi_{1}\left(y^{\prime}\right) \phi_{2}\left(z^{\prime}\right) e^{-y^{\prime 2} / 2 s}\left(e^{-\frac{\left(z^{\prime}-y^{\prime}\right)^{2}}{2(t-s)}}+e^{-\frac{\left(z^{\prime}+y^{\prime}\right)^{2}}{2(t-s)}}\right) \\
& =\mathbb{E}\left[\phi_{1}\left(\left|B_{s}\right|\right) \phi_{2}\left(\left|B_{t}\right|\right)\right] .
\end{aligned}
$$

Using a similar estimate as the one in (5), we get 


$$
\lim _{n \rightarrow+\infty} \mathbb{E}\left[\phi_{1}\left(X_{n}(s)\right) \phi_{2}\left(X_{n}(t)\right)\right]=\mathbb{E}\left[\phi_{1}\left(\left|B_{s}\right|\right) \phi_{2}\left(\left|B_{t}\right|\right)\right],
$$

which concludes the convergence of $\left(X_{n}\right)$ in two-dimensional marginal distribution to a reflected Brownian motion.

\subsection{Finite dimensional distributions}

The convergence of $d$-dimensional marginal distributions of $\left(X_{n}(t)\right)_{n \geq 1}$ for any $d \geq 2$ may be done by induction on $d$. Let us fix $n \geq 1, d \geq 3$, then reals $0<s_{1}<\cdots<s_{d}$ and $\phi_{1}, \ldots, \phi_{d}$ bounded and Lipschitz continuous real valued functions defined on $\mathbb{R}$.

Let $\kappa$ denote the first reflection time after $\left[n s_{1}\right]$, i.e., $\kappa=\kappa\left(n, s_{1}\right)=\min \{k>$ $\left.\left[n s_{1}\right]: X(k-1)+\xi_{k}<0\right\}$. We decompose $\mathbb{E}_{x}\left[\prod_{i=1}^{d} \phi_{i}\left(\frac{X\left(\left[n s_{i}\right]\right)}{\sigma \sqrt{n}}\right)\right]$ as

$$
\sum_{j=1}^{d-1} \sum_{k=\left[n s_{j}\right]+1}^{\left[n s_{j+1}\right]} \mathbb{E}_{x}\left[\prod_{i=1}^{d} \phi_{i}\left(\frac{X\left(\left[n s_{i}\right]\right)}{\sigma \sqrt{n}}\right) ; \kappa=k\right]+\mathbb{E}_{x}\left[\prod_{i=1}^{d} \phi_{i}\left(\frac{X\left(\left[n s_{i}\right]\right)}{\sigma \sqrt{n}}\right) ; \kappa>\left[n s_{d}\right]\right] .
$$

Then we can deal with the terms

$$
\mathbb{E}_{x}\left[\prod_{i=1}^{d} \phi_{i}\left(\frac{X\left(\left[n s_{i}\right]\right)}{\sigma \sqrt{n}}\right) ; \kappa=k\right] \quad \text { and } \quad \mathbb{E}_{x}\left[\prod_{i=1}^{d} \phi_{i}\left(\frac{X\left(\left[n s_{i}\right]\right)}{\sigma \sqrt{n}}\right) ; \kappa>\left[n s_{d}\right]\right]
$$

in the same ways as we do for $A_{1}$ and $A_{2}$, respectively.

More precisely, for each $1 \leq j \leq d-1$ and $k \in\left\{\left[n s_{j}\right]+1, \ldots,\left[n s_{j+1}\right]\right\}$, we write 


$$
\begin{aligned}
\mathbb{E}_{x}\left[\prod_{i=1}^{d} \phi_{i}\left(\frac{X\left(\left[n s_{i}\right]\right)}{\sigma \sqrt{n}}\right) ; \kappa=k\right] \\
=\sum_{k_{1}=0}^{\left[n s_{1}\right]-1} \sum_{l \geq 0} \sum_{y \geq 1} \sum_{z \geq 1} \sum_{w \geq 0} \mathbb{E}_{x}\left[\prod_{i=1}^{d} \phi_{i}\left(\frac{X\left(\left[n s_{i}\right]\right)}{\sigma \sqrt{n}}\right) ; r_{l}=k_{1}, X\left(k_{1}\right)=z,\right. \\
\left.z+\xi_{k_{1}+1} \geq 0, \ldots, z+\xi_{k_{1}+1}+\cdots+\xi_{k-2} \geq 0, z+\xi_{k_{1}+1}+\cdots+\xi_{k-1}=w, w+\xi_{k}=-y\right] \\
=\sum_{k_{1}=0}^{\left[n s_{1}\right]-1} \sum_{l \geq 0} \sum_{y \geq 1} \sum_{z \geq 1} \sum_{w \geq 0} \mathbb{E}_{x}\left[\prod_{i_{1}=1}^{j} \phi_{i_{1}}\left(\frac{z+\xi_{k_{1}+1}+\cdots+\xi_{\left[n s_{j}\right]}}{\sigma \sqrt{n}}\right)\right. \\
\quad \times \prod_{i_{2}=j+1}^{d} \phi_{i_{2}}\left(\frac{y+\xi_{k+1}+\cdots+\xi_{\left[n s_{j}\right]}}{\sigma \sqrt{n}}\right) ; r_{l}=k_{1}, X\left(k_{1}\right)=z, z+\xi_{k_{1}+1} \geq 0, \ldots, \\
\left.z+\xi_{k_{1}+1}+\cdots+\xi_{k-2} \geq 0, z+\xi_{k_{1}+1}+\cdots+\xi_{k-1}=w, w+\xi_{k}=-y\right] \\
=\sum_{k_{1}=0}^{\left[n s_{1}\right]-1} \sum_{z \geq 1} \Sigma_{k_{1}}(x, z) \sum_{y \geq 1} \sum_{w \geq 0} \mathbb{E}_{y}\left[\prod_{i_{2}=j+1}^{d} \phi_{i_{2}}\left(\frac{X\left(\left[n s_{j}\right]-k_{2}\right)}{\sigma \sqrt{n}}\right)\right] \\
\quad \times \mathbb{E}\left[\prod_{i_{1}=1}^{j} \phi_{i_{1}}\left(\frac{z+S\left(\left[n s_{j}\right]-k_{1}\right)}{\sigma \sqrt{n}}\right) \mid \tau^{S}(z)>k-k_{1}-1, z+S\left(k-k_{1}-1\right)=w\right] \\
\quad \times \mathbb{P}\left[\tau^{S}(z)>k-k_{1}-1, z+S\left(k-k_{1}-1\right)=w\right] \mathbb{P}\left[\xi_{1}=-w-y\right] .
\end{aligned}
$$

Now we can use the induction hypothesis and Corollary 2.5 in [5] to deal with the first and the second expectations.

\subsection{Tightness}

Recall that the modulus of continuity of a function $f:[0,1] \rightarrow \mathbb{R}$ is defined by

$$
w_{f}(\delta)=\sup _{t, s \in[0,1],|t-s|<\delta}|f(t)-f(s)| .
$$

It is clear that $w_{X}(\delta) \leq w_{S}(\delta)$. Using Theorem 7.3 in [3], the tightness of $X$ follows directly from the one of the classical random walk $(S(n))_{n \geq 0}$. We achieve the proof of Theorem 1, applying Theorem 7.1 in [3].

\section{Auxiliary proofs}

Proof of Lemma 7. By setting $h_{n}(y)=\sqrt{n} \mathbb{P}_{y}\left[r_{1}>n\right]$, the Markov property yields 


$$
\begin{aligned}
\widehat{\Sigma}_{n}(x, t, s) & =n \sum_{l \geq 0} \mathbb{E}_{X}\left[\mathbb{P}_{X\left(r_{l}\right)}\left[r_{1} \circ \theta^{r_{l}}>[n t]-[n s]\right] ; r_{l}=[n s]\right] \\
& =\frac{\sqrt{n}}{\sqrt{[n t]-[n s]}} \sqrt{n} \sum_{l \geq 0} \mathbb{E}_{X}\left[h_{[n t]-[n s]}\left(X\left(r_{l}\right)\right) ; r_{l}=[n s]\right] \\
& =\frac{1+o(n)}{\sqrt{s(t-s)}} \sqrt{[n s]} T_{[n s]}\left(h_{[n t]-[n s]}\right)(x) .
\end{aligned}
$$

Let us prove that $\sqrt{[n s]} T_{[n s]}\left(h_{[n t]-[n s]}\right)(x) \rightarrow \frac{1}{\pi}$ as $n \rightarrow+\infty$. Indeed,

$$
\left|\sqrt{[n s]} T_{[n s]}\left(h_{[n t]-[n s]}\right)(x)-\frac{1}{\pi}\right| \leq B_{1}(n)+B_{2}(n),
$$

with

$$
\begin{aligned}
& B_{1}(n)=\left|\sqrt{[n s]} T_{[n s]}\left(h_{[n t]-[n s]}\right)(x)-\frac{1}{\pi v(h)} v\left(h_{[n t]-[n s]}\right)\right|, \quad \text { and } \\
& B_{2}(n)=\frac{1}{\pi v(h)}\left|v\left(h_{[n t]-[n s]}\right)-v(h)\right| .
\end{aligned}
$$

By Lemma 1, it holds $0 \leq h_{n}(y) \leq C_{1} h(y)$, with $h(y)=O(y)$, so that the sequence $\left(h_{n}\right)_{n \geq 1}$ is bounded in $\mathcal{B}_{\alpha}$. Thus, Corollary 2 yields

$$
B_{1}(n) \leq(1+x)\left|\sqrt{[n s]} T_{[n s]}-\frac{1}{\pi v(h)} \Pi\right|_{\alpha}\left|h_{[n t]-[n s]}\right|_{\alpha} \longrightarrow 0 \quad \text { as } \quad n \rightarrow+\infty .
$$

Similarly, by Lemma 1 and the dominated convergence theorem,

$$
\lim _{n \rightarrow+\infty}\left|v\left(h_{[n t]-[n s]}\right)-v(h)\right|=0,
$$

so that $B_{2}(n) \longrightarrow 0$ as $n \rightarrow+\infty$.

Proof of Lemma 9. By setting $\tilde{h}_{n}(y)=n^{3 / 2} \mathbb{P}_{y}\left[r_{1}=n\right]$, the Markov property yields

$$
\begin{aligned}
\widetilde{\Sigma}_{n}(x, s, t) & =n^{2} \sum_{l \geq 0} \mathbb{E}_{x}\left[\mathbb{P}_{X\left(r_{l}\right)}\left[r_{1} \circ \theta^{r_{l}}=[n t]-[n s]\right] ; r_{l}=[n s]\right] \\
& =\frac{n^{3 / 2}}{([n t]-[n s])^{3 / 2}} \sqrt{n} \sum_{l \geq 0} \mathbb{E}_{x}\left[\tilde{h}_{[n t]-[n s]}\left(X\left(r_{l}\right)\right) ; r_{l}=[n s]\right] \\
& =\frac{1+o(n)}{\sqrt{s}(t-s)^{3 / 2}} \sqrt{[n s]} T_{[n s]}\left(\tilde{h}_{[n t]-[n s]}\right)(x) .
\end{aligned}
$$

By Corollary 1, it holds $0 \leq \tilde{h}_{n}(y) \leq C_{3} h(y)$, with $h(y)=O(y)$, so that the sequence $\left(\tilde{h}_{n}\right)_{n \geq 1}$ is bounded in $\mathcal{B}_{\alpha}$. We conclude as above to prove Lemma 7 . 


\section{References}

1. Afanasyev, V. I., Böinghoff, C., Kersting, G., Vatutin, V. A.: Limit theorems for weakly subcritical branching processes in random environment, Journal of Theoretical Probability, 25, No. 3, 703-732 (2012)

2. Afanasyev, V. I., Geiger, J., Kersting, G., Vatutin, V. A.: Criticality for branching processes in random environment, The Annals of Probability, 33, No. 2, 645-673 (2005)

3. Billingsley, P.: Convergence of Probability Measures, Willey, New York (1968)

4. Bolthausen, E.: On a Functional Central Limit Theorem for Random Walks Conditioned to Stay Positive, The Annals of Probability, 4, No. 3, 480-485 (1976)

5. Caravenna, F., Chaumont, L.: An invariance principle for random walk bridges conditioned to stay positive, Electron. J. Probab., 18, No. 60, 1-32 (2013)

6. Chow, Y. S., and Lai, T. L.: Moments of ladder variables for driftless random walks, Z. Wahrsch. Verw. Gebiete, 48, 253-257 (1979)

7. Doney, R. A.: Local behaviour of first passage probabilities. Probability Theory and Related Fields, 152 (3-4), 559-588 (2012)

8. Essifi, R., Peigné, M.: Return Probabilities for the Reflected Random Walk on $\mathbb{N}_{0}$. Journal of Theoretical Probability, 28, No. 1, 231-258 (2015)

9. Gouëzel, S.: Correlation asymptotics from large deviations in dynamical systems with infinite measure. Colloquium Mathematicum, 125, 193-212 (2011)

10. Iglehart, D. L.: Functional central limit theorems for random walks conditioned to stay positive, The Annals of Probability, 2, No. 4, 608-619 (1974).

11. Itô, K., and P. McKean H.P Jr.: Diffusion processes and their sample paths, Springer Science Business Media (2012)

12. Kemperman, J. H. B.: The oscillating random walk, Stoch. Process and Their Appl., 2, 1-29 (1974)

13. Kozlov, M.V.: On the asymptotic behavior of the probability of non-extinction for critical branching processes in a random environment, Theory Probab. Appl., 21, No. 4, 791-804 (1976)

14. Le Page, E., Peigné, M.: A local limit theorem on the semi-direct product of $\mathbb{R}^{*+}$ and $\mathbb{R}^{d}$, Annales de l'I.H.P. Probab. et Stat., 33, No. 2, 223-252 (1997)

15. Ngo, H-L., Peigné, M.: Limit theorem for perturbed random walks. To appear in Theory of Stochastic Processes (2020)

16. Peigné, M., Woess, W.: Stochastic dynamical systems with weak contractivity I. Strong and local contractivity, Colloquium Mathematicum, 125, 1-54 (2011)

17. Peigné, M., Woess, W.: On recurrence of a reflected random walk on the half line. http://arxiv.org/abs/ math/0612306 (2006) 